\title{
The management of peanut allergy
}

\author{
Katherine Anagnostou, ${ }^{1}$ Andrew Clark $^{2}$
}

${ }^{1}$ Department of Paediatric Allergy, Guy's and St Thomas' Hospitals NHS Foundation Trust, London, UK

${ }^{2}$ Department of Medicine, University of Cambridge, Addenbrooke's Hospital, Cambridge, UK

\section{Correspondence to}

Dr Andrew Clark, Department of Medicine, University of Cambridge, Box 157, Addenbrooke's Hospital, Cambridge CB2 OQQ, UK atclark@doctors.org.uk

Received 16 June 2014 Revised 4 August 2014 Accepted 6 August 2014 Published Online First 25 August 2014

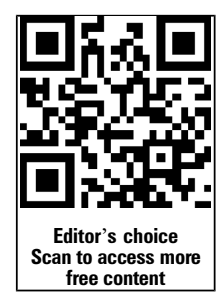

CrossMark

To cite: Anagnostou K, Clark A. Arch Dis Child 2015;100:68-72.

\section{ABSTRACT}

Peanut allergy is common and can be a cause of severe, life-threatening reactions. It is rarely outgrown like other food allergies such as egg and milk. Measures aiming to reduce its prevalence via maternal avoidance during pregnancy and lactation, or delayed introduction into the diet, have failed to show any benefit. Peanut allergy has a significant effect on the quality of life of sufferers and their families due to dietary and social restrictions, but mainly stemming from fear of accidental peanut ingestion. The current management consists of strict avoidance, education and provision of emergency medication. Families find avoidance challenging as peanut is hidden in various food products. Despite the fact that food labelling has improved, with a legal obligation to declare certain food allergens (including nuts) in prepacked products, it still causes confusion and does not extend to cross-contamination. In an effort to address issues of safety at school, a lot of work has been undertaken to better care for peanut-allergic children in that environment. This includes training of school staff on how to recognise and treat allergic reactions promptly. Recent developments in the management of peanut allergy, such as immunotherapy, have shown some promise as an active form of treatment, but larger studies are required to further investigate safety and efficacy.

\section{BACKGROUND}

Peanut allergy was once rare, but it is now the most common cause of fatal food-allergic reactions. ${ }^{1}$ The prevalence has increased steadily over the past decade, mostly in the Western world, the disease currently affecting $1-2 \%$ of children in the UK..$^{2-4}$ A study in the Isle of Wight showed a twofold increase in reported peanut allergy and a threefold increase in sensitisation, in two birth cohorts of children over a period of 7 years. ${ }^{4}$ A similar trend has been noticed in the USA. Sicherer $e t$ al reported a significant increase in peanut allergy in children, from $0.4 \%$ in 1997 to $0.8 \%$ in 2002 to $1.4 \%$ in 2008 , determined by a random digit dial telephone survey; this is equal to a 3.5 -fold increase within a period of 11 years. $^{5-7}$ In Asian countries, peanut allergy has a much lower prevalence. ${ }^{8}$

In two different case series of fatal food-allergic reactions published in 1992 and 2001, respectively, peanut is a common cause. ${ }^{1} 9$ Sampson et al described 13 cases of fatal or near-fatal anaphylactic reactions to foods in children and adolescents 2-17 years old over a period of 14 months. ${ }^{1}$ Fatal reactions are generally rare, but the possibility results in significant anxiety as they can occur due to accidental peanut ingestion at any time. Accidental reactions are common as peanuts can be hidden in various foods or contaminate meals in restaurants. ${ }^{10} 11$ Peanut-allergic individuals experience higher levels of anxiety and increased awareness that their condition can be fatal; they also feel that they have less control over their disease compared with diabetic children. ${ }^{12}$ Parents of peanut-allergic children also present high levels of stress, mainly due to their child's risk of death and constant dietary restrictions. ${ }^{13}$

In contrast to other food allergies such as egg and milk-both of which are usually outgrown in the majority of patients-only a small percentage of children are expected to outgrow their peanut allergy (approximately 20\% based on published studies, though resolution in clinical practice is rare). ${ }^{14-17}$

\section{THE IMPORTANCE OF CORRECT DIAGNOSIS}

Making the correct diagnosis of peanut allergy is of paramount importance. A typical history of a hypersensitivity reaction to peanut following ingestion, in combination with a positive skin prick test or peanut specific IgE, would point towards a diagnosis of peanut allergy. Typical symptoms include urticarial, angioedema, nausea, abdominal pain, vomiting, wheezing and breathlessness, occurring soon after ingestion. If peanut has not been ingested, but there is a positive test result to peanut, measured because of other food allergies or atopic disease, it is important to remember that a significant proportion will be sensitised (have specific IgE), but have no clinical allergy. A carefully conducted oral challenge may be required in unusual circumstances where there is disagreement between the clinical picture and specific IgE tests. ${ }^{18}$ Recent food allergy guidance by the National Institute for Health and Care Excellence (NICE) provides useful information on when to consider and how to make the correct diagnosis of peanut allergy (NICE CG116).

Recently, tests for specific IgE to individual components of peanut protein (Ara h1-11) have been introduced to help with the diagnosis of peanut allergy, although they are not widely available yet. An evaluation of $\mathrm{IgE}$ binding to different peanut proteins (components) can add important information into making the diagnosis of peanut allergy and potentially differentiate between sensitisation and clinical reactivity. ${ }^{19}$ Initial studies showed that component resolved diagnostics could improve the specificity of peanut allergy testing. Ara h 2, the major peanut allergen, is a seed storage protein, resistant to both heat and digestion. In patients with suspected peanut allergy, IgE to Ara h 2 has been shown to be a more specific and more accurate diagnostic test than crude peanut-specific $\operatorname{IgE}$ and may provide clinicians with a way to minimise the need for peanut challenges. ${ }^{20}{ }^{21}$ Children with typical peanut allergy were found to have $\mathrm{IgE}$ that 
binds predominantly to Ara h 2 and Ara h $6 .^{22}$ However, patterns of binding to peanut protein vary significantly in different geographical areas, and the clinical usefulness of these tests is still to be determined. ${ }^{23}$ The Immuno Solid phase Allergy Chip screening test measures over a hundred allergen components from a single blood sample, with the aim of determining patterns of reactivity. The role of these so-called component-resolved diagnostic tests in clinical practice has not yet been definitively established.

\section{PRIMARY PREVENTION}

According to recommendations published in 1998 by the Department of Health (which have now been withdrawn), it was stated that "pregnant women who are atopic or have an atopic partner may wish to avoid eating peanuts during pregnancy and lactation. Infants with a family history of atopy, should be exclusively breastfed for 4-6 months and should avoid peanuts until 3 years.". ${ }^{25}$ This advice was based on the assumption that peanut sensitisation occurs as a result of in utero exposure or via breast feeding. These recommendations were subsequently withdrawn as there was not sufficient evidence to support that maternal avoidance has any benefits in preventing the development of peanut allergy in infants. ${ }^{26} 27$ Currently, pregnant women are not actively discouraged from eating peanuts during pregnancy and lactation.

The association between consumption of peanuts and tree nuts during pregnancy by non-allergic mothers was investigated by a recent prospective study. The authors actually reported that higher consumption of peanuts and tree nuts by the mothers peri-pregnancy was associated with a lower risk of peanut and tree nut allergy in the newborn infant. ${ }^{28}$

Environmental exposure to peanut via the oral route appears to play an important role in the development of tolerance. High-level environmental exposure (presence and/or consumption of peanut in various forms in the home environment) in infancy, in the absence of oral exposure, is associated with peanut sensitisation. High levels of oral exposure, on the other hand, lead to tolerance irrespective of environmental exposure. $^{29}$ Early consumption of peanuts in infancy, as well as consumption of frequent and high doses of peanut protein, was also found to be associated with a low prevalence of peanut allergy, possibly due to induction of oral tolerance. ${ }^{30}$

In practical terms, the above evidence suggests that even in high-risk children, the delayed introduction of peanut may result in an increased risk for the development of peanut allergy. However, data from ongoing studies such as Learning Early About Peanut allergy (LEAP) are needed to better understand whether early peanut introduction can actively help reduce the risk of peanut allergy. The LEAP study involves 640 high-risk children, enrolled when aged 4-11 months, examining the effect of early peanut consumption on the risk of developing peanut allergy. ${ }^{31}$ Each child was randomly assigned to follow either avoidance or consumption of an age-appropriate peanut snack three times a week. The proportion of each group that develops peanut allergy by 5 years of age will be compared to determine which approach works best for preventing peanut allergy. $^{31}$

\section{EDUCATION}

The current standard of care for the management of peanut allergy is to advise strict peanut avoidance and provide emergency medication (antihistamines, $\beta 2$ agonists, injectable intramuscular adrenaline). ${ }^{32}$ In the UK, the prescription of adrenaline devices is generally recommended for patients with previous anaphylaxis, significant reactions to trace amounts of peanut or significant asthma. Other factors, such as limited access to emergency medical care and the patient's age (adolescents are considered at higher risk for life-threatening allergic reactions), may also be taken into account.

Educating the families on how to avoid accidental ingestion, recognise and treat allergic reactions promptly when they occur, forms the cornerstone of current management for peanut allergy. In the majority of cases of fatal and near-fatal reactions to peanuts, patients were unaware that the food they consumed contained peanut, indicating that attempts at avoidance are often unsuccessful. ${ }^{1}$ Case series of fatalities in children have shown that of the six children that died, three deaths $(50 \%)$ followed a reaction to peanuts. Only two of the six patients that died received adrenaline within an hour of ingesting the allergen and none of them received it when the initial symptoms appeared. ${ }^{9}$ Bock et al reported 32 deaths of individuals 2-33 years of age over a 5 -year period, $63 \%$ of which are attributed to peanuts. Only $10 \%$ of these individuals had adrenaline available for use at the time of their reaction. ${ }^{1}$

Patients and their families must be educated on how to recognise and treat allergic reactions, when to ask for help and how to use their emergency medication. A single visit to a multidisciplinary allergy clinic can improve families' abilities to manage allergic reactions considerably and reduce the number of subsequent accidental reactions. ${ }^{33}$ A comprehensive management plan including verbal and written advice on nut avoidance as well as training in recognition and treatment of allergic reactions, with the addition of a written management plan, can be effective in reducing both severity and number of future reactions. ${ }^{33-35}$

\section{AVOIDANCE}

\section{Risk of accidental reactions}

Families of peanut-allergic children have shown poor knowledge of how to avoid food allergens, treat food allergy emergencies and use an adrenaline autoinjector. ${ }^{33}$ Avoidance can be difficult as peanuts are widely used in many different foods. There is also the risk of contamination of foods with peanut protein during the manufacturing process. Labelling can be inadequate or misinterpreted by families and caregivers. ${ }^{36}$

It is not surprising, therefore, that accidental reactions are common. Annual incidence rates for accidental reactions vary between studies; $15 \%$ in a group of 567 UK patients with nut allergy referred to their allergy clinic and followed up annually, ${ }^{35} 55 \%$ over 5 years in a cohort of 102 peanut-allergic children in the $\mathrm{USA}^{37}$ and $75 \%$ over a period of 14 years in another US study. ${ }^{14}$ Further, nearly $1 / 3$ of nut-allergic children cannot recognise the nut to which they are allergic. This lack of recognition puts them at increased risk for unintentional ingestion. $^{38}$

It is also important to highlight the unpredictability of future accidental reactions even if the initial reaction on presentation was considered mild. A longitudinal study of a population of children who developed peanut allergy before the age of 4 years revealed that of the children with initial non-life-threatening reactions, $44 \%$ had at least one potentially life-threatening reaction during follow-up. ${ }^{39}$

\section{AVOID INDEX NUT OR ALL NUTS}

A significant proportion of peanut-allergic children (25-50\%) will also report allergy to tree nuts. ${ }^{18} \mathrm{~A}$ common initial presentation is allergy to peanut or a single tree nut, but subsequently, children may develop multiple nut allergies. An observational, 
cross-sectional study of 784 children in the UK has shown that by 2 years of age at least 19\% were multisensitised and $2 \%$ multiallergic. Increasing proportions were exposed to multiple nut types with increasing age (23\% at 2 years to $73 \%$ by 10 years) and greater proportions were multisensitised (19\% at 2 years to $86 \%$ at $5-14$ years) and multiallergic (2\% at 2 years to $47 \%$ at 14 years). ${ }^{40}$

Many allergists recommend complete avoidance of all tree nuts in a child with peanut allergy. This advice is supported by the possible risk of cross-contamination between various nuts and also problems with labelling of food products. In addition, children are often unable to recognise the nut they are allergic to and this puts them at increased risk of accidental reactions. Complete avoidance of all nuts would prevent this. ${ }^{38}$

On the other hand, the avoidance of nut consumption in early life may be associated with an increased risk of development of new nut allergies. It is possible that the introduction of certain nuts (to which the child is not allergic to) may prevent the development of further nut allergies and at the same time expanding the repertoire of the child's diet. More studies are needed in this area in order to make the correct recommendations on nut consumption.

\section{ADVISORY LABELS}

Dietary restrictions appear to play an important role in the reduced quality of life in peanut-allergic children. Simple tasks such as eating in restaurants can be frightening due to the risk of food contamination with peanut. Shopping, on the other hand, can be time consuming (due to constant checking of food labelling), frustrating and limited because of a growing number of products having a label of 'may contain peanut' even if they seem unrelated to peanut or nuts. 121341

Product labels should be clear and accurate in order to convey the necessary information to allergic consumers. Unfortunately, food labelling still remains ambiguous and in a questionnaire survey of 184 food-allergic children, labels including 'this product does not contain any nuts but is made in a factory that uses nuts', 'cannot guarantee is nut free' and 'may contain traces of nuts' were avoided by only around 50\% of parents of nut-allergic children. Previous allergic reaction to nut products had no bearing on outcome. ${ }^{42}$

Hefle et al have shown that the likelihood of a product with an advisory warning label actually containing peanut residue was $4.7 \%$; however, up to $7 \%$ of products showed some evidence of peanut residue. Despite the fact that the vast majority of foods with advisory labels do not contain sufficient allergen to trigger an allergic reaction, the risk is not trivial and the authors concluded that it is advisable that consumers avoid all such items. ${ }^{43}$

It is now a legal requirement, in many countries around the world, for specific food allergens to be disclosed when they are ingredients in prepacked foods. There are currently 14 food allergens that must be disclosed. Current legislation does not cover cross-contamination. ${ }^{414}$ At the end of 2014, new legislation (the EU Food Information for Consumers Regulation 1169/ 2011) will be introduced that will require food businesses to provide allergy information on food sold unpackaged, in catering outlets, deli counters, bakeries and sandwich bars. ${ }^{41}$

A recent study investigating the advice provided by 239 British healthcare professionals to allergy sufferers revealed that only $38 \%$ of health professionals recommended complete avoidance of foods with advisory labels to nuts (when no nut was listed in the ingredients), whereas $22 \%$ advised their patients that no avoidance was necessary. Only $14 \%$ stressed the importance of carrying an adrenaline device and rescue medication. More cautious advice was provided to those with asthma, previous history of anaphylaxis or previous reaction to a trace amount of the relevant allergen. ${ }^{45}$

Although there is no clear consensus on what advice should be provided to allergy sufferers, it is important for the patients and their families that the issue is addressed as part of an individual management plan for nut allergy. Complete avoidance would result in fewer reactions overall, but on the other hand, a more relaxed approach would result in significantly wider food choices. More data are required to show whether less stringent avoidance measures are likely to put patients at significant risk of severe reaction.

\section{SCHOOL/AIR TRAVEL}

Allergy affects at least one quarter of European schoolchildren, and $20 \%$ of food-allergic reactions have been noted to occur at school. ${ }^{46}$ Recently, a taskforce position paper was published by the European Academy of Allergy and Clinical Immunology (EAACI) on the management of allergic children at school in order to ensure that such children are protected. The document clearly outlines the rights of the allergic child and the role of various stakeholders (family, medical professionals, school staff and policymakers). It also gives a detailed description of action points for all children with allergic disease at school and outlines the importance of providing individualised anaphylaxis management plans for children at risk. Close cooperation of families, doctors and school staff is necessary to ensure that food-allergic children are well cared for in the school environment. All school staff should be trained in the early recognition and treatment of allergic reactions, including the use of emergency medication. ${ }^{46}$

Safety during commercial air travel is an important issue for peanut-allergic children and their families. Various airlines have different policies on how to deal with food allergies and also the provision of high-risk foods. A US study investigating selfreported allergic reactions to nuts and seeds aboard commercial airliners showed that $9 \%$ of food-allergic individuals reported reactions, mostly to peanuts. Worryingly, only 29\% of these individuals informed a flight attendant of their reaction. In addition, when information was requested from customer services regarding flying with food allergies, it was found to be incomplete or inaccurate. ${ }^{47}$ More work is required in order to better inform the industry on high-risk foods and ensure the safety of passengers with peanut and other food allergies.

\section{FUTURE RESEARCH}

\section{Immunotherapy}

An area of intense research interest in the future management of peanut allergy is peanut oral immunotherapy (OIT), which has shown promise as a form of active treatment. The administration of small but increasing doses of peanut protein (an average peanut contains $160 \mathrm{mg}$ of peanut protein although variations occur up to $260 \mathrm{mg}$ depending on size) to children that are allergic peanut has been shown to increase their reactive threshold and enable them to eat varying amounts of peanut without reactions. ${ }^{48-50}$

A phase II, randomised-controlled, crossover trial of peanut OIT recently published in the Lancet investigated the role of peanut OIT in 99 children inclusive of all severities of peanut allergy. In the active group, $84 \%$ were desensitised to $800 \mathrm{mg}$, whereas 24 of 39 OIT participants were successfully desensitised to $1400 \mathrm{mg}$ of peanut protein (approximately 10 roast peanuts). Subjects who successfully completed the OIT study 
protocol had a significant 25 -fold increase of their peanut threshold, and their caregivers had a significant improvement in quality of life. Adverse effects in most were mild and easily treatable. ${ }^{50}$

Larger studies are needed to further improve safety and efficacy of this form of treatment. Patients will need to balance the frequent reactions occurring during immunotherapy, with the risk of severe reactions due to accidental ingestion and the possibility of successful desensitisation, by the end of treatment. Long-term tolerance following immunotherapy is still an area that requires further investigation.

Trials are also underway using immunotherapy by different routes such as epicutaneous and sublingual. Other treatment options are also under investigation such as the use of adjuvants (anti-IgE) in combination with OIT.

\section{Summary points}

- Peanut allergy is common and can cause severe, life-threatening reactions. It has a significant effect in quality of life.

- Avoidance of peanut consumption during pregnancy and lactation failed to reduce the prevalence of peanut allergy. Early introduction of peanut may actually promote tolerance and reduce the risk of peanut allergy.

- Current management consists of strict avoidance of peanut, provision of emergency medication and educating the families on how to recognise and treat allergic reactions when they occur.

- Peanut avoidance is challenging and accidental reactions are common.

- Food labelling, although improved, can still be confusing and ambiguous for peanut-allergic children and their caregivers.

- Future developments of active management of peanut allergy include peanut oral immunotherapy.

Contributors The first draft was prepared by KA. AC and KA jointly edited and rewrote subsequent versions.

Competing interests KA: none. AC: Patent applicant for oral immunotherapy regime.

Provenance and peer review Commissioned; externally peer reviewed.

\section{REFERENCES}

1 Bock SA, Muñoz-Furlong A, Sampson HA. Fatalities due to anaphylactic reactions to foods. J Allergy Clin Immunol 2001;107:191-3.

2 Hourihane JO, Aiken R, Briggs R, et al. The impact of government advice to pregnant mothers regarding peanut avoidance on the prevalence of peanut allergy in United Kingdom children at school entry. J Allergy Clin Immunol 2007;119:1197-202

3 Pereira B, Venter C, Grundy J, et al. Prevalence of sensitization to food allergens, reported adverse reaction to foods, food avoidance, and food hypersensitivity among teenagers. J Allergy Clin Immunol 2005;116:884-92.

4 Grundy J. Rising prevalence of allergy to peanut in children: data from 2 sequential cohorts. J Allergy Clin Immunol 2002;110:784-9.

5 Sicherer SH, Munozfurlong A, Burks AW, et al. Prevalence of peanut and tree nut allergy in the US determined by a random digit dial telephone survey. I Allergy Clin Immunol 1999:103:559-62.

6 Sicherer SH, Muñoz-Furlong A, Sampson HA. Prevalence of peanut and tree nut allergy in the United States determined by means of a random digit dial telephone survey. A 5-year follow-up study. J Allergy Clin Immunol 2003;112:1203-7.

7 Sicherer SH, Muñoz-Furlong A, Godbold JH, et al. US prevalence of self-reported peanut, tree nut, and sesame allergy: 11-year follow-up. J Allergy Clin Immunol 2010;125:1322-6.
8 Shek LP-C, Cabrera-Morales EA, Soh SE, et al. A population-based questionnaire survey on the prevalence of peanut, tree nut, and shellfish allergy in 2 Asian populations. J Allergy Clin Immunol 2010;126:324-31.e7.

9 Sampson HA, Mendelson L, Rosen JP. Fatal and near-fatal anaphylactic reactions to food in children and adolescents. N Engl J Med 1992;327:380-4.

10 Vadas $\mathrm{P}$, Perelman B. Presence of undeclared peanut protein in chocolate bars imported from Europe. J Food Prot 2003;66:1932-4.

11 Schäppi GF, Konrad V, Imhof D, et al. Hidden peanut allergens detected in various foods: findings and legal measures. Allergy 2001;56:1216-20.

12 Avery NJ, King RM, Knight $\mathrm{S}$, et al. Assessment of quality of life in children with peanut allergy. Pediatr Allergy Immunol 2003;14:378-82.

13 Primeau MN, Kagan R, Joseph L, et al. The psychological burden of peanut allergy as perceived by adults with peanut allergy and the parents of peanut-allergic children. Clin Exp Allergy 2000;30:1135-43.

14 Bock SA, Atkins F. The natural history of peanut allergy 1. J Allergy Clin Immunol 1989:83:900-4.

15 Hourihane JO, Roberts SA, Warner JO. Resolution of peanut allergy: case-control study. BMJ 1998;316:1271-5.

16 Skolnick HS, Conover-Walker MK, Koerner CB, et al. The natural history of peanut allergy. J Allergy Clin Immunol 2001;107:367-74.

17 Ho MHK, Wong WHS, Heine RG, et al. Early clinical predictors of remission of peanut allergy in children. J Allergy Clin Immunol 2008;121:731-6.

18 Sicherer SH, Sampson HA. Peanut allergy: Emerging concepts and approaches for an apparent epidemic. J Allergy Clin Immunol 2007;120:491-503.

19 Sicherer SH, Wood RA. Advances in diagnosing peanut allergy. J Allergy Clin Immunol Pract 2013;1:1-13.

20 Lieberman JA, Glaumann S, Batelson S, et al. The utility of peanut components in the diagnosis of IgE-mediated peanut allergy among distinct populations. J Allergy Clin Immunol Pract 2013;1:75-82.

21 Nicolaou N, Poorafshar M, Murray C, et al. Allergy or tolerance in children sensitized to peanut: prevalence and differentiation using component-resolved diagnostics. J Allergy Clin Immunol 2010;125:191-7.e1-13.

22 Blanc F, Adel-Patient K, Drumare M-F, et al. Capacity of purified peanut allergens to induce degranulation in a functional in vitro assay: Ara $\mathrm{h} 2$ and Ara $\mathrm{h} 6$ are the most efficient elicitors. Clin Exp Allergy 2009;39:1277-85.

23 Venter C, Hasan Arshad S, Grundy J, et al. Time trends in the prevalence of peanut allergy: three cohorts of children from the same geographical location in the UK. Allergy 2010;65:103-8.

24 Lauer I, Dueringer N, Pokoj S, et al. The non-specific lipid transfer protein, Ara h 9 , is an important allergen in peanut. Clin Exp Allergy 2009;39:1427-37.

25 Committee on toxicity of chemicals in food consumer products and the environment. Review of advice on peanut avoidance. 2008.

26 Greer FR, Sicherer SH, Burks a W. Effects of early nutritional interventions on the development of atopic disease in infants and children: the role of maternal dietary restriction, breastfeeding, timing of introduction of complementary foods, and hydrolyzed formulas. Pediatrics 2008:121:183-91.

27 Fleischer DM, Spergel JM, Assa'ad AH, et al. Primary prevention of allergic disease through nutritional interventions. J allergy Clin Immunol Pract 2013;1: 29-36.

28 Frazier AL, Camargo CA, Malspeis S, et al. Prospective study of peripregnancy consumption of peanuts or tree nuts by mothers and the risk of peanut or tree nut allergy in their offspring. JAMA Pediatr 2013;168:156-62.

29 Fox AT, Sasieni P, du Toit G, et al. Household peanut consumption as a risk factor for the development of peanut allergy. J Allergy Clin Immunol 2009;123: 417-23.

30 Du Toit G, Katz Y, Sasieni P, et al. Early consumption of peanuts in infancy is associated with a low prevalence of peanut allergy. J Allergy Clin Immunol 2008;122:984-91.

31 Du Toit G, Roberts G, Sayre PH, et al. Identifying infants at high risk of peanut allergy: the Learning Early About Peanut Allergy (LEAP) screening study. J Allergy Clin Immunol 2013;131:135-43.e1-12.

32 Sampson HA. Food allergy. Part 2: diagnosis and management. J Allergy Clin Immunol. 1999:103:981-9.

33 Kapoor S, Roberts $G$, Bynoe $Y$, et al. Influence of a multidisciplinary paediatric allergy clinic on parental knowledge and rate of subsequent allergic reactions. Allergy 2004:59:185-91.

34 Ewan PW, Clark AT. Efficacy of a management plan based on severity assessment in longitudinal and case-controlled studies of 747 children with nut allergy: proposal for good practice. Clin Exp Allergy 2005;35:751-6.

35 Ewan $\mathrm{P}$, Clark A. Long-term prospective observational study of patients with peanut and nut allergy after participation in a management plan. Lancet 2001;357:111-5

$36 \mathrm{Yu} J \mathrm{~W}$, Kagan R, Verreault $\mathrm{N}$, et al. Accidental ingestions in children with peanut allergy. J Allergy Clin Immunol 2006;118:466-72.

37 Sicherer SH, Burks AW, Sampson HA. Clinical features of acute allergic reactions to peanut and tree nuts in children. Pediatrics 1998;102:e6-e6.

38 Ferdman RM, Church JA. Mixed-up nuts: identification of peanuts and tree nuts by children. Ann Allergy Asthma Immunol 2006;97:73-7. 
39 Vander Leek TK, Liu AH, Stefanski K, et al. The natural history of peanut allergy in young children and its association with serum peanut-specific lgE. J Pediatr 2000;137:749-55.

40 Clark AT, Ewan PW. The development and progression of allergy to multiple nuts at different ages. Pediatr Allergy Immunol 2005;16:507-11.

41 Aware B. "May Contain"Labelling-The Consumer's Perspective. food.gov.uk [Internet]. [cited 2014 May 9]; http://www.food.gov.uk/multimedia/pdfs/ maycontainreport.pdf

42 Noimark L, Gardner J, Warner JO. Parents' attitudes when purchasing products for children with nut allergy: a UK perspective. Pediatr Allergy Immunol 2009;20:500-4.

43 Hefle SL, Furlong TJ, Niemann L, et al. Consumer attitudes and risks associated with packaged foods having advisory labeling regarding the presence of peanuts. J Allergy Clin Immunol 2007;120:171-6.

44 Turner PJ, Kemp AS, Campbell DE. Advisory food labels: consumers with allergies need more than "traces" of information. BMJ 2011;343.
45 Turner PJ, Skypala IJ, Fox AT. Advice provided by Health Professionals regarding precautionary allergen labelling. Pediatr Allergy Immunol 2014;25:290-2.

46 Muraro A, Clark A, Beyer $K$, et al. The management of the allergic child at school: EAACI/GA2LEN Task Force on the allergic child at school. Allergy 2010;65: 681-9.

47 Comstock SS, DeMera R, Vega LC, et al. Allergic reactions to peanuts, tree nuts, and seeds aboard commercial airliners. Ann Allergy Asthma Immunol 2014;101:51-6.

48 Blumchen $\mathrm{K}$, Ulbricht $\mathrm{H}$, Staden $\mathrm{U}$, et al. Oral peanut immunotherapy in children with peanut anaphylaxis. J Allergy Clin Immunol 2010;126:83-91.e1.

49 Hofmann AM, Scurlock AM, Jones SM, et al. Safety of a peanut oral immunotherapy protocol in children with peanut allergy. J Allergy Clin Immunol; 2009;124:286-91, 291.e1-6.

50 Anagnostou K, Islam S, King Y, et al. Assessing the efficacy of oral immunotherapy for the desensitisation of peanut allergy in children (STOP II): a phase 2 randomised controlled trial. Lancet 2014;383:1297-304. 